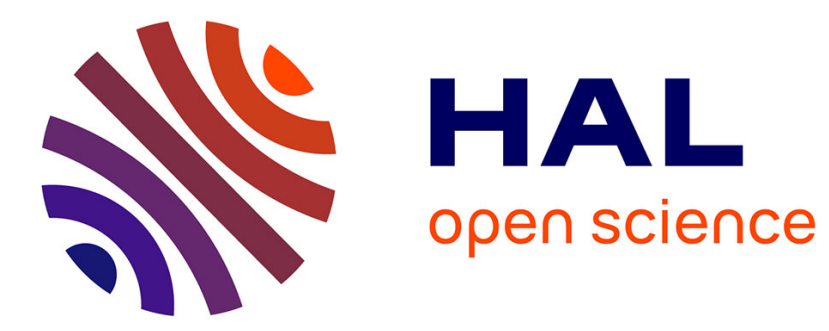

\title{
Mises en scène de Shakespeare dans Le Digamma d'Yves Bonnefoy
}

Pascale Drouet

\section{To cite this version:}

Pascale Drouet. Mises en scène de Shakespeare dans Le Digamma d'Yves Bonnefoy. Revue d'histoire littéraire de la France, 2014, 114 (4), pp.933-946. 10.3917/rhlf.144.0933 halshs-01840786

\section{HAL Id: halshs-01840786 \\ https://shs.hal.science/halshs-01840786}

Submitted on 23 Aug 2018

HAL is a multi-disciplinary open access archive for the deposit and dissemination of scientific research documents, whether they are published or not. The documents may come from teaching and research institutions in France or abroad, or from public or private research centers.
L'archive ouverte pluridisciplinaire HAL, est destinée au dépôt et à la diffusion de documents scientifiques de niveau recherche, publiés ou non, émanant des établissements d'enseignement et de recherche français ou étrangers, des laboratoires publics ou privés. 


\title{
MISES EN SCENNE DE SHAKESPEARE DANS LE DIGAMMA D'YVES BONNEFOY
}

Pascale Drouet

\author{
Presses Universitaires de France | «Revue d'histoire littéraire de la France »
}

2014/4 Vol. 114 | pages 933 à 946

ISSN 0035-2411

ISBN 9782130629283

Article disponible en ligne à l'adresse :

https://www.cairn.info/revue-d-histoire-litteraire-de-la-france-2014-4-page-933.htm

\section{Pour citer cet article :}

Pascale Drouet, « Mises en scène de Shakespeare dans Le Digamma d'Yves Bonnefoy », Revue d'histoire littéraire de la France 2014/4 (Vol. 114), p. 933-946. DOI 10.3917/rhlf.144.0933

Distribution électronique Cairn.info pour Presses Universitaires de France.

(C) Presses Universitaires de France. Tous droits réservés pour tous pays.

La reproduction ou représentation de cet article, notamment par photocopie, n'est autorisée que dans les limites des conditions générales d'utilisation du site ou, le cas échéant, des conditions générales de la licence souscrite par votre établissement. Toute autre reproduction ou représentation, en tout ou partie, sous quelque forme et de quelque manière que ce soit, est interdite sauf accord préalable et écrit de l'éditeur, en dehors des cas prévus par la législation en vigueur en France. Il est précisé que son stockage dans une base de données est également interdit. 


\title{
MISES EN SCÈNE DE SHAKESPEARE DANS LE DIGAMMA D'YVES BONNEFOY
}

\author{
Pascale Drouet*
}

Dans son recueil intitulé L'heure présente, publié en 2011, Yves Bonnefoy consacrait deux textes en prose à Hamlet dans lesquels il explorait la possibilité d'une mise en scène renouvelée de la célèbre tragédie, un Hamlet porté non à la scène mais à la montagne, requérant des acteurs et des spectateurs mobiles, comme en perpétuel mouvement parmi des parois rocheuses, des torrents, des moraines, des terrasses herbeuses, des sapins enneigés, des chemins de traverse ( Première ébauche d'une mise en scène d'Hamlet » et « Hamlet en montagne »).

Dans Le Digamma, son recueil récent paru fin 2012, Yves Bonnefoy nous propose d'assister à l'ébauche d'une mise en scène tout à fait originale d'Othello résultant d'un « étrange projet ${ }^{1}$ », celui d'un théâtre d'ombres («Pour mettre en scène Othello »). Mais, auparavant, le poète revient vers certains «événements incompréhensibles ${ }^{2}$ » touchant les répétitions d'un ancien Hamlet (« Dieu dans Hamlet »). Ainsi Yves Bonnefoy poursuit-il son dialogue avec Shakespeare, avec, toujours, sa prédilection attentive pour ces grandes figures féminines que sont Ophélie et Desdémone.

Dans sa préface de 2001 à sa traduction d'Othello, Yves Bonnefoy mettait en rapport Hamlet et Othello et s'en expliquait ainsi : « Ce qui était le "sub-plot" dans Hamlet est dans Othello maintenant la totalité de l'œuvre, avec d'ailleurs presque le même drame : l'homme insultant la femme qu'il aime et qu'il n'aime pas, la menaçant, la faisant mourir, mourant lui-

* Université de Poitiers.

1. Yves Bonnefoy, "Pour mettre en scène Othello », in Le Digamma, Paris, Galilée, coll. « Lignes fictives », 2012, p. 61.

2. Yves Bonnefoy, « Dieu dans Hamlet», in Le Digamma, op. cit., p. 9.

RHLF, 2014, n 4, pp. 933-946 
même ${ }^{3}$ ». Les tragédies témoignent, à son sens, du même souci ontologique : Shakespeare s'y livre à l'étude d'une conscience de soi, de la capacité de ses personnages à être au monde et à ce qui sape cette capacité - le « to be or not to be » d'Hamlet; le «I am not what I am » de Iago.

Ce qui rapproche ces textes sur les mises en scène d'Hamlet et d'Othello dans Le Digamma, c'est, me semble-t-il, d'une part, l'interrogation à laquelle se livre Bonnefoy sur la sensation d'étouffement, la présence de la malédiction («Dieu dans Hamlet») et du mal (« Pour mettre en scène Othello »), sur la façon de les appréhender (l'échec d'un dieu envieux) ou de les dépasser (persistance du visage humain), et, d'autre part, la question ontologique de notre être-là en tant qu'existant, notre capacité d'incarnation (l'excellence d'un acteur dans son rôle, mais aussi l'acquiescement de l'être humain à sa finitude charnelle), c'est-à-dire notre présence et notre résistance par une émotion qui garde intacte son intensité (la tête penchée de Desdémone), par l'espoir persistant de l'inespéré et par le risque du partage (la capacité de s'unir à d'autres; la main qui se tend).

" DIEU DANS HAMLET » : ÉTOUFFER, RÔDER, ÊTRE

Dans « Dieu dans Hamlet», comme dans "Première ébauche d'une mise en scène d'Hamlet », le spectacle quitte la salle traditionnelle pour s'exporter ailleurs, afin de répondre au «grand désir inquiet d'élargir la scène $»^{4}$ du metteur en scène et d'expérimenter un espace scénique ouvert, à même la nature, car il s'agit d'appréhender autrement le texte et de se mettre en quête de tous ses sens possibles. Or les répétitions ne se déroulent pas comme prévu. Le narrateur commence par retracer les « bizarreries ${ }^{5} »$, les « étranges événements ${ }^{6}$ », qui n’ont cessé de ponctuer cette expérience nouvelle, puis cherche à en proposer une interprétation rétrospective.

\section{Sensation d'étouffement}

Tout s'origine dans un «même désir d'accroissement de l'espace scénique $^{7} »$. L'air paraît manquer à Polonius; Ophélie semble vouloir s'enfuir. De l'ensemble des comédiens, Yves Bonnefoy nous dit : « Ils aimaient rester assez loin les uns des autres. On eût dit qu'ils voulaient laisser vide le

3. Yves Bonnefoy, «La tête penchée de Desdémone », in William Shakespeare, Othello, Préface et traduction d'Yves Bonnefoy, Édition bilingue, Paris, Gallimard, coll. " Folio théâtre ", 2001, p. 59.

4. Id.

5. Ibid., p. 12.

6. Ibid., p. 25.

7. Ibid., p. 9. 
centre du plateau ${ }^{8}$. \ Si l'espace leur est devenu vital, ce n'est pas tant dans l'espoir de l'investir scéniquement que de le ressaisir avec son vide. La sensation d'étouffement qui s'éprouve dans la salle traditionnelle, au point de générer une anxiété et un mal-être menant aux larmes, conduit le metteur en scène à « quitter le théâtre, et même [à] aller loin, très loin, dans la sombre campagne de ce pays ", dans " un champ, sous le vaste ciel bas de ce bout du monde $»^{9}$. Les comédiens, comme le metteur en scène, aspirent à s'affranchir des codes scéniques, à se mouvoir dans un espace non saturé par l'humain et ses constructions, à ouvrir comme à l'infini leur espace de jeu et leur liberté d'expression.

Ce déplacement audacieux signe l'originalité de la mise en scène : bruits et échos naturels créent des interférences inédites dans les dialogues, invitant ainsi à leur prêter une écoute différente et à les repenser, invitant aussi à de nouvelles modalités du dire - jusqu'où pourra-t-on faire porter sa voix ? Jusqu'où pourra-t-on l'entendre et la reconnaître dans ses altérations ? Hamlet, on le sait, est la tragédie de Shakespeare la plus portée à la scène. Il s'agit ici de chercher à entendre autrement les mots tant dits du texte shakespearien, comme par espoir de leur redonner une saveur quasi originelle, primitive, mystérieuse : «Les voix reviendraient en écho de la falaise voisine, avec des retards, elles se mêleraient, on se prendrait à aimer les harmoniques qui troubleraient dans les mots d'alors le son et même le sens ${ }^{10}$. » Le metteur en scène d'Hamlet rejoint ici celui d'Othello en son désir de « mieux laisser voir en sa profondeur difficile un possible étouffé dans les représentations ordinaires ${ }^{11} »$.

La préface qu'Yves Bonnefoy avait écrite suite à sa traduction d'Hamlet éclaire le projet du metteur en scène de «Dieu dans Hamlet». Dans « Readiness, ripeness : Hamlet, Lear », le poète rappelle que la tragédie du prince du Danemark est traversée par la fracture entre l'époque médiévale et les temps modernes. C'est l'intégration de l'homme dans le monde qui se perd à la charnière des $\mathrm{XVI}^{\mathrm{e}}$ et $\mathrm{XVII}^{\mathrm{e}}$ siècles. Yves Bonnefoy précise :

Quand une pensée du tout, de l'unité, et de celle-ci comme vie, comme présence, réglait tous les rapports qu'on pouvait entretenir avec les réalités particulières. Chacune faisait ainsi partie d'un ordre, précisément défini, qui faisait d'elle à son tour une présence, une sorte d'âme éveillée à soi et au monde parmi les autres douées de la même vie, et lui assurait un sens, dont il n'y avait pas à douter. La conséquence la plus importante, et heureuse, de ce fait d'un ordre et du sens, c'est que la personne humaine, qui se savait un élément de ce monde et s'en croyait parfois même le centre, n'avait pas à douter non plus de son être propre, de sa qualité d'absolu. Quels

8. Ibid., p. 10.

9. Id.

10. Ibid., p. 10-11.

11. Yves Bonnefoy, « Pour mettre en scène Othello », in Le Digamma, op. cit., p. 62. 
que fussent les hauts et les bas de son existence, où intervenait le hasard, elle pouvait et devait en distinguer son essence, qui préservait une étincelle divine ${ }^{12}$.

Les tergiversations d'Hamlet, son incapacité à passer à l'acte, sa philosophie pessimiste reflètent cette perte et en disent l'ampleur. L'écart des conceptions qu'ont Hamlet père et Hamlet fils, des regards qu'ils portent sur le monde, se fait l'écho de cette fracture. La tragédie témoigne de l'ordre qui se fragmente, de la dispersion du sacré, voire de sa disparition, de la difficulté à se redéfinir en tant qu'être humain et à trouver sa place dans un univers semblant dépourvu de transcendance et de sens. Pour qui a compris qu'Hamlet est traversé par cette fracture et que le drame se joue donc dans « un monde déstructuré, des vérités désormais partielles, concurrentes, contradictoires », quoi de plus naturel que de soustraire sa mise en scène à l'unité d'un théâtre traditionnel ?

Or, la sensation d'étouffement récurrente dans «Dieu dans Hamlet » n'est-elle pas à entendre aussi au sens ontologique du terme ? Sans intuition originale, le projet du metteur en scène ne serait qu'un ajout à peine signifiant à une série déjà saturée d'adaptations scéniques. Les acteurs incarnant Ophélie et Polonius semblent échouer dans leur capacité à être au monde, à être pour l'autre. Ophélie-actrice aime « rester assise à l'écart, les yeux dans le vide ${ }^{13} \gg$; Polonius-acteur, lui, semble ne prendre vie qu'à travers les humbles objets qu'il souhaite mêler à la mise en scène et quand il paraît avec sa fille, celle-ci fuit dès qu'elle le peut l'emprise paternelle pour aller sangloter au loin - rapport père-fille malheureux tant dans la vie que dans la tragédie. Leur manque à être ne peut, alors, être qu'exacerbé par leur personnage respectif. Ophélie, comme Desdémone, est niée par celui qu'elle aime et en meurt. Aussi, le lieu n'y pouvant rien, la sensation d'étouffement persiste-t-elle en pleine nature, au point d'en inquiéter le narrateur : « Je ne dirais rien des autres bizarreries de ces premiers jours, elles nous amusaient, nous troublaient, un peu, mais ne nous inquiétaient pas, et nous cherchions à les oublier. Mais tout autre chose ce fut quand des sortes de spasmes saisirent, au point de les étouffer, des acteurs qui l'instant d'avant s'efforçaient paisiblement de bien interpréter une scène ${ }^{14}$. » Le malaise prend alors l'allure d'une inquiétante malédiction.

\section{Présence-absence du dieu envieux}

Lors de la scène cruciale où, observé par Claudius et Polonius, Hamlet rejette Ophélie avec violence, l'actrice est soudain comme possédée : «Une

12. Yves Bonnefoy, « Readiness, ripeness : Lear, Hamlet », in Théâtre et poésie : Shakespeare et Yeats, Paris, Mercure de France, 1998, p. 69-70.

13. Yves Bonnefoy, «Dieu dans Hamlet », in Le Digamma, op. cit., p. 10.

14. Ibid., p. 12. 
immense agitation s'empara de tout son corps, sa gorge se serra, rendant ses mots inintelligibles, des sanglots la secouèrent, ses bras se dressèrent, ils semblaient s'arracher d'elle, flotter un instant dans l'espace sous le ciel redevenu clair, puis elle s'écroula, dans la boue, et resta prostrée, toujours tremblante ${ }^{15}$. » Cette manifestation irrationnelle se présente comme le surgissement d'une inquiétante étrangeté. Un phénomène similaire se produit lorsque l'acteur professionnel relate la mort d'Hécube découvrant le corps sanglant de Priam. Comme Yves Bonnefoy qui l'écoute à cet instant précis, l'acteur est saisi :

Mais le cri ! Le cri de bête blessée à mort, non, pire encore, que le comédien poussa quand il dut rappeler celui qu'Hécube elle-même avait jeté à la face des dieux sur les murailles de Troie ! Ce cri monta d'un gouffre comme je n'imaginais pas qu'il y en eût un dans aucune existence humaine. Il enveloppa le sanglot de la vieille femme, se fondit en lui, le fit éclater, l'emportant haut dans le ciel, le préservant, cependant : c'était bien un cri qu'expliquait le texte, celui de cet homme qui chancelait, mais c'était quelque chose de plus encore, et d'autre, de tout autre, tout autant une souffrance terrible que, puis-je oser me risquer à parler ainsi, l'évidence, en cette voix rauque, d'une extraordinaire douceur. Comme avec Ophélie quelques jours plus tôt j'eus l'impression, nous eûmes tous l'impression, qu'une puissance d'au-delà toute mesure s'était glissée dans un être de moindre taille, le violentant. Mais, cette fois encore, que c'était plus pour s'identifier à lui que par désir de l'anéantir ${ }^{16}$.

Pour reprendre les termes de Jean-Louis Chrétien, le cri est ici «l'effraction soudaine, au beau milieu du cours des choses, d'un affect violent et comme $\operatorname{pur}^{17} \gg$. Le poète comprend rétrospectivement que ces comédiens, comme en transe à leur insu, sont le lieu de la manifestation d'une ventriloquie divine, ce que confirme l'épisode d'Hamlet se mettant, étonnement, à jouer du flageolet et en tirant «cette musique venue d'ailleurs que de lui ${ }^{18} » \ldots$ avant qu'on ne retrouve, un matin, son corps étrangement calciné. Alors, pris d'effroi, le metteur en scène et son équipe abandonnent les répétitions et ce projet qui semble attirer sur lui la malédiction. C'était, pourtant, d'Hamlet et non de la « pièce écossaise » qu'il s'agissait ${ }^{19}$.

Après avoir, des années plus tard, ressaisi ces événements par l'écriture, le narrateur tente de leur trouver un sens, se refusant à y voir simplement « une longue suite de hasards ${ }^{20}$ ». Contrairement au personnage d'Hamlet, il refuse de se satisfaire de «l'accueil des choses comme elles viennent»,

15. Ibid., p. 14 .

16. Ibid., p. 16-17.

17. Jean-Louis Chrétien, "Poétique du cri», in Les Cahiers de l'Herne, n 93 "Yves Bonnefoy », Paris, Éditions de l'Herne, 2010, p. 31.

18. Ibid., p. 19.

19. "Macbeth has already been regarded by actors as unlucky, and many accidents have befallen performers in the play, some of them fairly serious. Actors call it "the Scottish play", refusing to mention its name, especially on stage » (Marjorie Garber, Shakespeare and Modern Culture, New York, Anchor Books, 2008, p. 88).

20. Yves Bonnefoy, « Dieu dans Hamlet », in Le Digamma, op. cit., p. 19. 
de «l'acceptation du hasard $»^{21}$; il n'est pas prêt à tout accepter « avec ironie et indifférence ${ }^{22} »$. Il repense alors à un dieu fictif se rêvant à l'image de l'homme, un dieu qui aurait un inconscient et qui découvrirait donc que « quelqu'un en lui, qu'il ne savait pas, troublait ses intentions, enténébrait sa pensée, déconcertait son intelligence ${ }^{23} »$. Car, nous explique le poète, " ce qu'on nomme une création, à quelque niveau que ce soit, ce n'est jamais que de l'écriture, c'est-à-dire une place laissée, et peut-être la principale, aux pensées inconscientes de celui ou de celle qui écrit ${ }^{24} »$. Dieu se manifeste alors selon une sorte de présence-absence ; il se met à " rôder autour de ces lieux où on cherche à comprendre Hamlet ${ }^{25} »$; il entreprend de se glisser dans les acteurs pour mieux saisir ce qu'est l'humain avec ses débordements émotionnels et son inconscient. Ainsi Dieu continue-t-il de chercher. Les théologiens l'ont entendu « qui rôde / Dans le cri de l'oiseau blessé, dans le jappement / De la bête $\operatorname{prise}^{26}$ »; il est pour eux « la bête furtive au cœur maçonné de nuit ${ }^{27}$ ». Dans son poème « L'encore aveugle », Yves Bonnefoy dit les limites d'un dieu impuissant à ressentir l'étendue palette des émotions humaines :

Espérer,

Il sait que c'est plus que lui. Attendre,

Il sait que c'est plus que lui,

Apercevoir au loin, crier,

Se précipiter les bras ouverts, dans les larmes,

Il sait que c'est plus que lui ${ }^{28}$.

C'est pourtant ce qu'il tente encore dans « Dieu dans Hamlet». Or, à nouveau, Dieu échoue à « creuser en eux [les hommes] la voie de son éva$\operatorname{sion}^{29}$ ", à s'incarner, à ressentir pleinement la capacité proprement humaine d'écouter ce qui bat à la surface du monde, comme celle de se perdre dans ses propres profondeurs avant de les faire surgir en une émotion intense. Dieu découvre « que l'émotion qui bouleverse Ophélie, il ne peut la vivre ; que celle qui étrangle l'acteur qui pense à Hécube, il ne la comprend même $\operatorname{pas}^{30} »$. Celui qui, au final, étouffe dans sa condition, ce n'est pas l'homme mais Dieu, car, ce que ce dernier éprouve au moment même où il échoue à

21. Yves Bonnefoy, «Readiness, ripeness: Lear, Hamlet», in Théâtre et poésie : Shakespeare et Yeats, op. cit., p. 76.

22. Id.

23. Yves Bonnefoy, « Dieu dans Hamlet », in Le Digamma, op. cit., p. 22.

24. Ibid., p. 21.

25. Ibid., p. 23.

26. Yves Bonnefoy, «L'encore aveugle », in Les Planches courbes, Paris, Mercure de France, 2001, p. 111.

27. Ibid., p. 112.

28. Ibid., p. 110.

29. Yves Bonnefoy, « Dieu dans Hamlet », op. cit., p. 24.

30. Id. 
s'incarner, c'est que « son inhibition absolue, vaste comme la nuit, lui fait rentrer les mots dans la gorge quand il croit faire sienne la simple humaine douleur $^{31}$ ». Pour Jean-Louis Chrétien, « le désappointement de ce Dieu fictif est ce qui, par contraste, révèle la joie et la beauté de notre finitude ${ }^{32} »$. Échec de Dieu ou grandeur de l'acteur qui, éphémère, ressent et transmet le texte de Shakespeare qui embrasse toute l'humanité ?

Ce qui me semble important, ce n'est pas tant de s'interroger sur la crédibilité de l'explication du poète que de remarquer que son entreprise interprétative se démarque radicalement de l'attitude du personnage d'Hamlet dans la tragédie éponyme, de sa philosophie pessimiste face à la perte des repères, à l'insuffisance du sens, à l'évidement du cosmos. Yves Bonnefoy rejette chez Hamlet ce « célibat de l'âme, assumé comme un dernier signe, de défi désirant, qu'on fait à ce Dieu qui s'est retiré de son verbe ${ }^{33} »$. Face à ce qui interroge et semble défier le sens et notre faculté d'interprétation dans « Dieu dans Hamlet », les étranges événements relatés -, il nous propose plutôt de nous tourner vers « un ordre encore, universel, profond, celui de la vie qui, comprise, reconnue dans ses formes simples, aimée, acceptée, peut faire refleurir de son unité, de sa suffisance - ainsi l'herbe repousse dans les ruines - notre condition d'exilés du monde de la Promesse $\gg^{34}$.

\section{Présence}

Dans « Sortie du jardin sous la neige », deuxième texte du Digamma, Yves Bonnefoy se penche sur l'histoire d'une autre malédiction, célèbre : la fuite d'Adam et Ève chassés du paradis. Or les épreuves sans nombre imposées par « celui qui maudissait ${ }^{35}$ " s'estompent avec l'arrivée d'impalpables flocons ; la neige, « cette amie inconnue ${ }^{36}$ », met à distance la malédiction. Ève et Adam s'éprouvent à nouveau comme êtres au monde en sentant des flocons de neige leur effleurer la peau.

À la fin de « Dieu dans Hamlet », le narrateur ne peut s'empêcher de se demander si son explication ne relève pas de la fable. Or il sait qu'il lui arrive de sentir cette présence-absence : «Quand j'écris, [...], je sens si fort peser sur moi un regard inquiet, jaloux, j'éprouve si clairement que quelqu'un est là, qui rôde autour du langage, je comprends même si bien que les mots que j'emploie désirent plus que ce qu'ils expriment ! Quelle émotion, quelquefois ! Je me mets debout, je regarde ici et là, non, personne ${ }^{37}$. »

31. Ibid., p. 25 .

32. Jean-Louis Chrétien, «Poétique du cri », in Les Cahiers de l'Herne, n 93, p. 35.

33. Yves Bonnefoy, «Readiness, ripeness: Lear, Hamlet », in Théâtre et poésie : Shakespeare et Yeats, op. cit., p. 77.

34. Ibid., p. 85.

35. Yves Bonnefoy, « Sortie du jardin sous la neige », in Le Digamma, op. cit., p. 30.

36. Id.

37. Yves Bonnefoy, « Dieu dans Hamlet », in Le Digamma, op. cit., p. 25. 
Que fait alors le poète ? Il se détourne de ce dieu incapable d'incarnation, prend un chemin de montagne et s'en va tutoyer les pierres « plutôt plates et souvent rondes de ce pays », les pierres « d'ici $»^{38}$ :

Pierre, je te regarde longtemps. Je vois sur toi, sous ces belles couleurs, de fines entailles enchevêtrées qui pourraient être des signes. Je tente de ne pas douter de ce que je sais qui est vrai : qu'elles n'en sont pas. Et qu'il vaut de n'aimer en toi, pierre d'ici, que ta capacité de t'unir à d'autres qui permet quelquefois que tienne debout, pour un moment, une voûte ${ }^{39}$.

N'y entend-on pas comme un écho aux propos sur la pierre que tenait le poète dans « Les tombeaux de Ravenne ${ }^{40}$ »? N'est-ce pas aussi une invite à nous méfier de notre propension à l'interprétation et, donc, à revenir au contact du réel en ce qu'il a de plus simple ? En cela, la fin de « Dieu dans Hamlet» est peut-être à mettre en regard de «Voix dans le bruit de la pluie » où la rêverie interprétative crée des espaces vertigineux dans lesquels s'abîmer. C'est la réalité d'une présence palpable, ramenée à sa simplicité, qui le sauve. Il se tourne vers sa compagne à demi endormie : « Existes-tu, toi, au moins ? lui demande-t-il, en la serrant dans ses bras. Mais oui, lui répond-elle, ne m'entends-tu pas te parler? De ses lèvres il cherche ces lèvres qui sont les mots qui suffisent ${ }^{41}$. " Ce qui fait sens, ce qui fait exister au sens plein du terme, qu'est-ce sinon notre capacité de nous unir à d'autres ? À une transcendance divine présente-absente, Yves Bonnefoy préfère l'immanence d'une existence humaine, une pierre que l'on ramasse, un corps que l'on étreint. Le mot " voûte » qui clôt le texte ne renvoie pas, pour lui, aux sphères célestes, mais à une construction humaine, à un bâtir ensemble, à une première pierre ${ }^{42}$.

Ainsi, par l'attitude qui est la sienne et la pensée qu'il nous livre dans le dernier paragraphe de « Dieu dans Hamlet », Yves Bonnefoy nous confirme que la fonction nouvelle de la poésie, en réponse à la fracture des temps modernes que Shakespeare, selon lui, avait clairement perçue, lui aussi l'a faite sienne : « Se souvenir, [...] espérer, [...] chercher par elle-même, [...]

38. Ibid., p. 26.

39. Id.

40. Voir Yves Bonnefoy, "Les tombeaux de Ravenne », in L'Improbable et autres essais, suivi de Un rêve fait à Mantoue, Paris, Gallimard, coll. «Folio essai », 1992, et notamment p. 29 (« L’Égypte affirme par la pierre que le seul avenir de l'homme est dans ce monde physique. Ainsi font à Ravenne les sarcophages du $\mathrm{VI}^{\mathrm{e}}$ siècle »).

41. Yves Bonnefoy, «Voix dans le bruit de la pluie », in Le Digamma, op. cit., p. 52.

42. La voûte qu'il se remémore dans un autre texte, "Bibliothèques savantes », est celle d'une chapelle devenue la bibliothèque souterraine du Clark Institute, d'un lieu de culte devenu lieu d'étude. Et s'il s'égare parfois sous des voûtes métaphoriques, ce ne sont pas celles d'un dieu créateur mais celles de son inconscient (Yves Bonnefoy, "Les œuvres de l'inconscient ", in Le Digamma, op. cit., p. 45). 
faire apparaître ce qui se cache dans les formes du quotidien sous les dissociations, les aliénations, de la science ou de la culture ${ }^{43}$. »

\section{"POUR METTRE EN SCÈNE OTHELLO ": L'ÉCHEC DE LA DÉSINCARNATION}

Cet avant-dernier texte du Digamma s'ouvre avec le souvenir qui hante le metteur en scène d'Othello : celui des peurs enfantines créées par ces jeux d'ombres chinoises auxquels il participait, enfant, et qui faisaient naître « des êtres qui paraissaient effrayés, reculant devant un autre qui avançait, le loup, disait-on, sa gueule ouverte ${ }^{44} »$. L'anxiété se faisait angoisse quand, seul dans sa chambre le soir, il voyait « tout près de lui, sur la paroi que touchait son lit, se hérisser l'ombre portée de l'oreiller, quelque chose d'énigmatique, de fantastique, une masse noire qui bougeait, comme sous l'effet d'une vie qui eût étouffé dans sa profondeur ${ }^{45} »$. Cet oreiller n'évoque-t-il pas celui qui, criminel, étouffe l'innocente Desdémone dans la tragédie de Shakespeare ? La comparaison « comme sous l'effet d'une vie qui eût étouffé dans sa profondeur» pourrait, en effet, évoquer les soubresauts faibles, presque déjà soumis, de Desdémone, ce personnage qui, tout comme celui d'Ophélie auquel il se fond dans le poème « L'heure présente ${ }^{46}$ », n'a cessé de retenir l'attention de Bonnefoy - ce n'est pas un hasard si sa préface à sa traduction d'Othello s'intitule « La tête penchée de Desdémone » (2001).

Ce souvenir n'est autre, en fait, que la genèse du projet original du metteur en scène dans son rapport aux acteurs, projet qui consiste à « ne pas s'occuper de ce qu'ils font de leur jeu sur scène pour ne plus penser qu'aux ombres qu'ils pourraient jeter sur un $\operatorname{mur}^{47} »$. Quant aux voix, elles seraient « grossies par un haut-parleur » et elles « erreraient dans l'espace sombre, portant le texte fatal $»^{48}$.

\section{La tentative du théâtre d'ombres}

Il faut au préalable souligner qu'Othello est, pour le metteur en scène, la tragédie noire par excellence, celle de la «perfidie », de « la ténèbre », du « mal absolu », du « mal sans motivation, rien que soi $»^{49}$, qui dévoile peu

43. Yves Bonnefoy, "Readiness, ripeness: Lear, Hamlet », in Théâtre et poésie : Shakespeare et Yeats, op. cit., p. 85.

44. Yves Bonnefoy, « Pour mettre en scène Othello », op. cit., p. 57.

45. Ibid., p. 58.

46. Voir « L'heure présente », in Yves Bonnefoy, L'Heure présente, Paris, Mercure de France, 2011, pp. 83-84.

47. Yves Bonnefoy, « Pour mettre en scène Othello », op. cit., p. 60.

48. Id.

49. Ibid., p. 59. 
à peu « la logique dévastatrice du traitre ${ }^{50}$ » et amène tout lecteur ou spectateur à se poser la question: «Va-t-on désespérer de la condition humaine $^{51}$ ? »Il veut s'employer à rendre cette noirceur. Or, voici que le théâtre d'ombres de son enfance lui revient en mémoire. Grâce à ce dispositif, le visage de l'acteur pourra se réduire à une découpe, à une caricature de l'humain, suggérant ainsi « le fond animal de la vie humaine, sa rapacité implacable, la matière qui prédomine dans ce qui semble l'esprit ${ }^{52} »$. À son sens, « le théâtre d'ombres opère à même niveau que l'idée pessimiste que semble énoncer la tragédie de Shakespeare ${ }^{53} »$. Car, ajoute-t-il plus loin, « l'ombre portée ne connaît pas le visage, elle ne sait rien de ce qui se joue en lui ${ }^{54} \gg$. En cela, l'ombre partage un trait propre à la sculpture : elle n'a pas de regard. Or, nous rappelle Hegel,

Ne regarde-t-on pas [...] tout homme d'abord et surtout dans les yeux, pour y trouver un repère, un point qui explique et résume lumineusement tout son aspect phénoménal, lequel se laisse saisir dans sa modalité la plus simple à partir du point d'unité du regard. Le regard est ce qu'il y a de plus chargé d'âme, la concentration de l'intimité et de la subjectivité sentante ; comme une poignée de main, et plus rapidement encore, le regard de l'œil permet à l'homme de se mettre à l'unisson avec son semblable ${ }^{55}$.

Toute intériorité à laquelle le regard permettait d'accéder est ainsi niée, du moins le metteur en scène le croit-il au début de son expérimentation.

Le texte qu'on entendrait serait comme désincarné, "prononcé sans autre assise que soi ${ }^{56} »$, les affects gommés, les interprétations délavées. Or, le théâtre d'ombres échoue dans sa tentative d'épure de la noirceur humaine : « Hélas, l'expérimentateur de ce théâtre des ombres doit bien constater qu'il n'a pas réussi à donner champ libre au mal qu'il désire combattre : autrement dit, à retirer de ses griffes ce visage humain qu'il rêve d'une nature tout autre ${ }^{57}$. » Il n'y a, certes, plus de visage pour trahir la fébrilité d'une belle âme, mais les gestes, eux, en prolongent les traits, et notamment les gestes de Desdémone et d'Emilia - « un mouvement, une main qui se risque vers une autre ${ }^{58} »$. Yves Bonnefoy souligne cet échec :

le rayon de la lampe n'éradique pas de l'ombre des gestes de Desdémone l'émotion qui l'étreint mais aussi l'espoir qu'elle ne peut renoncer, et c'est catastrophique, cela, pour le projet du metteur en scène. Car celui-ci, puisque ces gestes-ci sont donc

50. Ibid., p. 63 .

51. Ibid., p. 60

52. Ibid., p. 61.

53. Id.

54. Ibid., p. 62.

55. Hegel, Cours d'esthétique II, trad. de Jean-Pierre Lefebvre et Veronika von Schenck, Paris, Aubier, 1996, p. 368.

56. Yves Bonnefoy, « Pour mettre en scène Othello », p. 62.

57. Ibid., p. 65.

58. Id. 
le visage encore, ne peut que voir qu'il n'a pas réussi à séparer ce qu'il rêve une transcendance de cette triste réalité que le théâtre d'ombres a vocation de faire paraître ${ }^{59}$.

Que fait alors le metteur en scène ? Il renonce aux acteurs et recourt à « une autre famille d'ombres portées », celle des mannequins - « formes minimales », « quatre membres de bois articulés sur rotules», « têtes ovoïdes sans traits marqués $»^{60}$. Ces mannequins seront actionnés par des machinistes. Se retrouve, presque littéralement, l'effrayante idée qui parcourt la tragédie, à savoir que les personnages ne sont que des pantins dont Iago tire les ficelles.

Cette étrange expérimentation d'une adaptation dans laquelle les acteurs ont été remplacés par des mannequins sans vie, sans mouvement, pourait évoquer ces socles dépourvus de statues sur lesquels le poète attire notre attention dans « La tâche d'inexister ${ }^{61}$ ». Il s'agit, pour le metteur en scène, de « rompre, de rompre enfin, comme il l'avait espéré, avec toute vaine trace de l'ordinaire espérance ${ }^{62} »$. Or, le voici qui se met à voir des signes dessinés par l'action saccadée de ces " grandes choses inertes ${ }^{63}$ », c'est-àdire qu'il retrouve précisément ce qu'il avait voulu éviter : le combat « du signe et de la ténèbre ${ }^{64} \gg$. Le metteur en scène tente alors une ultime expérience, rejoignant en cela le metteur en scène d'Hamlet : il quitte le théâtre avec ses mannequins et part en quête de « champs parsemés de hautes pierres ». Toujours dans la même optique : «Espérer que projeter les ombres mêlées des mannequins et des pierres, celles-ci rien que des éboulis du rien du monde, en son hasard dépourvu de sens, pourrait enfin effacer de l'écran le souvenir du visage, laissant vivre dans ses bas-fonds de non-être la sorte de vérité qui le nie ${ }^{65}$. »

Or, force lui est de constater que, malgré « les amas de pierre » et « le pur brutal non-sens », l'humain ressurgit et que ressurgit, plus particulièrement, la tête penchée de Desdémone, «méditative, infiniment triste, espérante encore, supraterrestre $»^{66}$. On pourrait risquer l'hypothèse que la tête penchée de Desdémone qui se détache, de manière inattendue, d'un théâtre d'ombres mécanique est à mettre en rapport avec « le feu que courbait le vent de la $\operatorname{mer}^{67} »$, feu qui, incongru, prend parfois la place des statues sur

59. Ibid., p. 65-66.

60. Ibid., p. 66.

61. Voir « La tâche d'inexister », in Yves Bonnefoy, La Vie errante, suivi de Remarques sur le dessin, Paris, Gallimard, coll. « Poésie », 1993, p. 61.

62. Yves Bonnefoy, « Pour mettre en scène Othello », p. 67.

63. Id.

64. Ibid., p. 68.

65. Ibid., p. 69.

66. Ibid., p. 70.

67. Yves Bonnefoy, "La tâche d'inexister", in La Vie errante, suivi de Remarques sur le dessin, op. cit., p. 61. 
les socles vides. Patrick Née a souligné l'opposition entre cette vision du feu courbé de « La tâche d'inexister » et la réflexion du visiteur d'Amber dans L'Arrière-pays selon laquelle « exister montait droit comme une fumée que n'affectait aucun vent $»^{68}$. La tête penchée de Desdémone dirait alors le paradoxe de son « exister/inexister », modalité, nous rappelle Patrick Née, chère au poète ${ }^{69}$.

\section{La tête penchée de Desdémone}

Le metteur en scène voulait mettre l'accent sur l'aspect le plus désespérément sombre de l'âme « humaine » et, donc, éradiquer - peut-être pour, à un autre niveau, la préserver - l'intimité qui se dégage du visage. En réduisant ses acteurs à des ombres, il pensait avoir réglé la question du désir d'être, de l'être au monde, l'avoir évacuée comme Iago la bafoue dans la tragédie; en utilisant des mannequins, il s'imaginait pouvoir enfin se concentrer sur la mécanique du mal. Il est troublant de constater qu'en fait la vision qu'il essaie de projeter, dans ce qu'il croit être l'originalité de sa mise en scène, n'est autre que celle de Iago. Iago qui ne voit « rien partout que des marionnettes qui grincent à tous les vents » et dont le regard «n'a que désert devant soi »; Iago qui incarne « cette vision du non-sens, de la mauvaiseté de tout $\gg^{70}$.

Or, ce que le metteur en scène doit finalement se résoudre à comprendre, nous explique Yves Bonnefoy, c'est « que pour être soi, et accéder au grand sens, le visage humain ne doit nullement être dépossédé de sa chair ; que c'est dans la matière même du monde, aussi désastreuse soit-elle, que l'absolu, petite fleur, doit fleurir ${ }^{71} »$. Autrement dit, le metteur en scène a échoué à séparer radicalement 1 ' " immonde », et sa force de contamination, de la part d'humanité, si frêle et infime soit-elle, qui lui résiste.

Ce qu'Yves Bonnefoy continue à voir, lui, c'est la tête penchée de Desdémone qui, dans la tragédie, est l'aveu qu'elle est sur le point de renoncer, d'être vaincue par le mal. Elle penche la tête comme la servante de sa mère, Barbara, morte en chantant la chanson du saule, morte d'une trahison d'amour. Pour Yves Bonnefoy, « en se remémorant cette attitude de Barbara, Desdémone comprend à quel point, à quelle profondeur de son être, celle-ci renonçait à soi au moment même où pourtant elle chantait le saule, image

68. Yves Bonnefoy, L'Arrière-pays, augmenté d'une postafce, Paris, Gallimard, coll. " Poésie », [1972] 2005, p. 53.

69. Voir Patrick Née, Yves Bonnefoy penseur de l'image, ou les Travaux de Zeuxis, Paris, Gallimard, 2006, p. 236-238.

70. Yves Bonnefoy, «La tête penchée de Desdémone », in William Shakespeare, Othello, Préface et traduction d'Yves Bonnefoy, Édition bilingue, Paris, Gallimard, coll. " Folio théâtre ", 2001, p. 15.

71. Yves Bonnefoy, « Pour mettre en scène Othello », p. 70. 
du bien qu'on voudrait avoir ${ }^{72} \gg$. La tête penchée de Desdémone est indissociable de la chanson du saule et l'on comprend que cette chanson a, dans l'imaginaire du poète, la même force de transcendance que « la grande voix » à laquelle il rend un hommage si émouvant dans le texte qui clôt $L e$ Digamma, qu'elle est « à la fois réelle et imaginaire, autrement dit désignatrice de plus que ce monde bas de paroles toujours égocentriques $»^{73}$. L'on comprend que la tête penchée de Desdémone est au poète ce qu'est à la fille du potier de Corinthe le tracé de son amant sur le mur. Ce que la fille du potier va rendre, un peu à son insu, c'est bien plus que le souvenir d'une forme : «Celle qui simplement croyait vouloir comprendre une ligne dessine maintenant, quoi, une sorte d'arbre, des branches qui se gonflent de toutes parts, des oiseaux nombreux à s'envoler dans le ciel d'orage avec toutes sortes de faibles cris ${ }^{74}$. » Elle se retrouve, nous dit le poète, " à imaginer, à ressentir, à rêver ${ }^{75} »$. N'est-ce pas ce que fait Yves Bonnefoy quand il reconnaît la tête penchée de Desdémone ? Il ressent sa détresse, entend son appel à l'aide ; n'écrit-il pas, dans sa préface, que Desdémone « exprime qu'elle-même aurait bien besoin, dans sa situation présente, de l'affection et des soins qui vont aux tout petits êtres qui ont la tête encore penchée ${ }^{76}$ »? La «main qui se risque vers une autre ${ }^{77} »$, c'est aussi symboliquement celle du poète ; cette main qui a manqué à Desdémone pour qu'elle soit de ceux qui « ont foi en eux-mêmes et dans les autres, leurs proches, ce qui leur assure présence dans l'ici et le maintenant du monde », de ceux qui ont en eux « cette capacité d'être au monde $»^{78}$, c'est le poète qui la lui tend. Il lui prend la main, même, et cette main est bien entre les siennes.

Car, pour Yves Bonnefoy, la tête penchée de Desdémone ne se réduit pas à une simple découpe, elle a la force d'apparition, la force de présence d'un visage, elle produit une épiphanie semblable, elle " ouvre l'humanité ${ }^{79}$ ", selon le mot d'Emmanuel Levinas. On ne peut, ici, s'empêcher de penser à l'analyse du philosophe selon laquelle « la présence du visage - l'infini de l'Autre - est dénuement » et « appel venant de l'Autre pour m'appeler à la responsabilité », c'est-à-dire à un acte de « solidarité », de « fraternité $)^{80}$. Ainsi Desdémone existe-t-elle poétiquement par le désir d'Yves Bonnefoy de la faire advenir à l'être.

72. Ibid., p. 45.

73. Yves Bonnefoy, « La grande voix », in Le Digamma, op. cit., p. 75-76.

74. « Encore sur l'invention du dessin », p. 54.

75. Id.

76. Yves Bonnefoy, « La tête penchée de Desdémone », p. 45.

77. Yves Bonnefoy, « Pour mettre en scène Othello », p. 65.

78. Yves Bonnefoy, « La tête penchée de Desdémone », p. 16.

79. Emmanuel Levinas, Totalité et infini. Essai sur l'extériorité, Quatrième édition, Dordrecht, Kluwer Academic Publishers, coll. «Phaenomenologica », [1961] 1988, p. 188. 80. Ibid., p. 188, 189. 
Il parait naturel de mettre en relation ces deux textes du Digamma qui portent sur des mises en scène de tragédies. Or, outre qu'ils nous invitent à réfléchir à des façons novatrices d'adapter de grands textes shakespeariens, ils semblent former un diptyque inattendu. Comment ne pas penser que l'échec du metteur en scène d'Othello à désincarner à tout prix son spectacle, au prix de mannequins même, répond à l'échec de dieu à s'incarner, quitte à en ébranler fatalement l'humain, dans les personnages d'Hamlet ? Comment ne pas penser que le recours exclusif à des pantins inanimés qu'a le metteur en scène d'Othello est aussi vain qu'est stérile «la démesure inarticulée du $\mathrm{cri}^{81} \gg$ d'un dieu cherchant l'incarnation ?

Yves Bonnefoy se détourne de ces metteurs en scène et attire notre attention sur la voûte et sur le visage, tous deux perçus dans leur temporalité éphémère et pourtant demeurant essentiels en ce qu'ils disent ou appellent notre « capacité à [nous] unir à d'autres ${ }^{82}$ ». C'est là la véritable incarnation au sens où l'entend Yves Bonnefoy, incarnation que Patrick Née pense comme « échange vécu des existences, définissant une communauté humaine partagée ${ }^{83} »$. 